\title{
Shaikh, Anwar. Capitalism: Competition, Conflict and Crises. Nueva York: Oxford University Press, 2016, 1024 pp.
}

Muchas veces se suele dividir la literatura económica entre ortodoxa (o "mainstream") y heterodoxa (crítica). Mientras la literatura ortodoxa domina los libros de textos, donde modelos fundamentados matemáticamente desarrollan y hacen operativas premisas básicas como la competencia perfecta, la información completa, y la racionalidad de los agentes; la literatura heterodoxa suele componerse de visiones críticas a estas premisas, y recurre más que a la matemática, a la historia, la filosofía o al análisis político para mostrar la falsedad de la ortodoxia. Esta "división de trabajo" viene a ser un tácito equilibrio desigual donde la economía ortodoxa propone y la heterodoxa se limita a protestar, con pocas probabilidades de alterar el rumbo de la corriente principal.

El libro de Anwar Shaikh que reseño aquí plantea cambiar los términos mismos de este equilibrio, señalando que si realmente se quiere ir más allá del reclamo histérico para hacer una crítica que tenga un efecto material, es necesario proponer un fundamento alternativo que permita desarrollar instrumentos, leyes, y principios totalmente operativos y rigurosamente matematizados. Así, Capitalism es un libro sumamente ambicioso, ya que no solo plantea una metodología nueva y crítica de los enfoques existentes, sino que desarrolla su propuesta en todos los campos de la economía, abarcando tanto temas microeconómicos como macroeconómicos para constituirse como una teoría general del capitalismo (del mundo desarrollado). Esta ambición se corresponde con la biografía de su autor, natural de Pakistán y profesor de economía en la New School of Social Research de Nueva York por ya más de cuarenta años, institución desde la cual ha desarrollado una obra crítica en temas tan variados como teoría de la producción, historia del pensamiento, teoría del comercio, cálculo de cuentas nacionales, etc.; habiéndole tardado este libro en particular unos quince ańos. Una de las cualidades admirables de la propuesta del libro es que nos da todas las herramientas para evaluarlo con el mayor de los estándares, planteando con claridad sus tesis, y poniendo a disposición el álgebra, los métodos econométricos y el acervo estadístico usados (tanto en el apéndice de más de 300 páginas, como en un archivo on-line en realecon.org).

La propuesta de Shaikh se basa en evitar tanto el enfoque ortodoxo neoclásico como el enfoque heterodoxo, que atribuye básicamente a los enfoques post-keynesianos, y a otras escuelas críticas hacia la teoría neoclásica. El problema con estas últimas sería que no han renunciado aún al ideal de la teoría neoclásica, pues aunque tomen en cuenta la asimetría en la información y la presencia de monopolios, entre otras "imperfecciones”, estas siempre serán desviaciones de un supuesto estado ideal, que existió o existirá. Shaikh considera que tomar esta ruta para armar un esquema alternativo es inconsistente teóricamente ("no se puede hacer una teoría general de desviaciones") y se hace necesario optar por un rumbo que no haga ninguna referencia (ni positiva ni negativamente) a los supuestos neoclásicos. 
Shaikh llama a su teoría "competencia real", porque quiere partir de lo que se observa en el capitalismo realmente existente, la manera en que los actores (consumidores, firmas, bancos gobiernos, etc.) se desenvuelven. Como es evidente, este comportamiento real es sumamente complejo y sobredeterminado (por la historia, la etnia, la religión, el clima, la condiciones materiales, el género, etc.) y esta es una de las razones por las que el enfoque neoclásico ha legitimado el uso de sus abstracciones que simplifican este comportamiento a un agente representativo que permitiría explicar las leyes y patrones que se observan en el capitalismo (el crecimiento económico, los ciclos de negocios, auges y depresiones, etc.). Sin embargo, Shaikh demuestra que no es necesario plantear una microfundamentación idealizada como la neoclásica para representar tanto matemática como conceptualmente los patrones recurrentes del capitalismo. Más bien, otorga los elementos teóricos (cap. 3 y cap. 12) para comprender al capitalismo como un sistema complejo en donde precisamente la heterogeneidad y el desorden que lo constituyen generan propiedades emergentes que dan lugar a patrones recurrentes, ya que los agregados no están determinados por los microfundamentos. Esto permite que Shaikh, partiendo de una propuesta metodológica distinta y tomando en cuenta la heterogeneidad del comportamiento humano, pueda modelar las leyes fundamentales de la economía (oferta y demanda, rendimientos decrecientes, igualación de precios, salarios y tasas de ganancia) sin recurrir a los supuestos de competencia perfecta, información completa, comportamiento maximizador o expectativas racionales. Por lo tanto, si el enfoque neoclásico se amparaba en la intratable complejidad de lo real y sostenía que sus supuestos, aunque irreales, eran útiles para la predicción de patrones (el famoso argumento de Friedman), esta ya no sería una justificación válida, dado que Shaikh lograría explicar las mismas regularidades que el enfoque neoclásico además de, potencialmente, al partir de un marco distinto, acomodar hechos que la primera teoría no era capaz.

La teoría de la competencia real no es una teoría totalmente nueva, sino más bien el cuidadoso ensamble de una serie de componentes, en los que destaca y domina lo que Shaikh llama el pensamiento económico clásico, que incluye a Adam Smith (el concepto de la mano invisible), David Ricardo (teoría de precios y renta diferencial), Karl Marx (lucha de clases, la plusvalía como explotación, ejército industrial de reserva), Keynes y Kalecki (la demanda efectiva, la no-neutralidad del dinero), entre otros. Shaikh sostiene que la "derrota" de la economía política clásica por el enfoque neoclásico hacia finales del siglo XIX no estuvo justificada científicamente y fue más bien fruto de una operación ideológica (sostenida usualmente en la ventaja matemática de los neoclásicos) funcional para la defensa política del sistema capitalista que atravesaba las penurias de la crisis (la llamada depresión prolongada de 1873-1896). Sin embargo, la recuperación de los pensadores clásicos por parte de Shaikh no es un dogmatismo irreflexivo, en el sentido de sugerir que la economía contemporánea ya estaba explicada por Marx o Keynes — como hacen algunos de los textos heterodoxos-, pues constantemente se contrasta su relevancia con la evolución del capitalismo histórico. Antes que seguir una 
"línea correcta", Shaikh sintetiza a los distintos pensadores clásicos, a veces de manera algo polémica como, por ejemplo, con respecto a la teoría de valor trabajo de Marx en relación con la de Ricardo y Piero Sraffa (cap. 10). Asimismo, junto con la teoría clásica, Shaikh se abre a toda literatura que le ayude a explicar el objeto que está siguiendo, como puede ser la literatura de negocios que mostraría cómo realmente los empresarios compiten; la econofísica o teoría de la complejidad que permite modelar los patrones recurrentes; o la teoría de la reflexividad financiera de George Soros, que sirve para comprender la relación entre la especulación y los fundamentos económicos. A lo largo del libro (prácticamente cada capítulo), Shaikh reseña críticamente las teorías históricas sobre el tema relevante, plantea el enfoque clásico de manera formalizada, para luego verificarlo empíricamente y evaluar su utilidad.

La teoría clásica de Shaikh da la siguiente representación del capitalismo y su historia. Aunque la estructura de motivaciones sea compleja, el comportamiento motivado por el beneficio (profit motive) es el dominante y es lo que hace que las empresas pongan precios competitivos (en vez de ser precio-aceptantes) y busquen reducir costos, así como hace que los trabajadores se desplacen o se enfrasquen en luchas políticas para asegurarse el mejor salario. Aunque no hay concierto y planificación, estos movimientos generan, por el mismo proceso de la competencia, una igualación turbulenta (equalization by turbulent regulation) de los precios (tanto de mercancías como financieras), salarios y tasas de ganancia. Esta es la reinterpretación de la mano invisible de Smith por Shaikh, y hace énfasis en que todos estos procesos de igualación de las variables relevantes son más bien tendencias gravitatorias que crean distribuciones estables, antes que una igualdad, lo cual vuelve relevante la medición de los tiempos de ajuste y de las sensibilidades de las variables a cambios (lo que termina redefiniendo la separación entre corto plazo estático y largo plazo dinámico, Shaikh dirá: no hay corto plazo). Asimismo, si bien varios de estos patrones de igualación se vuelven visibles al mostrar los datos disponibles, en otros casos Shaikh ha necesitado usar métodos e instrumentos (en parte debido a que la producción estadística suele estar atada a supuestos y conceptos neoclásicos) para proponer nuevas medidas para variables como el capital (capitales reguladores) o los precios. En suma, la imagen agregada del capitalismo es la de un sistema regulado de manera caótica por la rentabilidad (profitability), que implica a su vez el salario, la tasa de crecimiento y el desempleo.

El libro es la comprobación de este nuevo marco tanto lógica como empíricamente, viendo en detalle hasta dónde puede servirnos para entender la teoría de la firma (cap. 4, cap. 7 y 8) y del consumidor, así como la dinámica de los precios relativos (cap. 5 y cap. 9), las finanzas (cap. 10) y el comercio internacional (cap. 11). Asimismo, Shaikh ofrece una novedosa teoría macroeconómica en la parte final del libro, entre las que destacan sus explicaciones clásicas del desempleo persistente y la dinámica de los salarios (cap. 14), de la inflación moderna (cap. 15) y de las crisis recurrentes del capitalismo (cap. 16). El capítulo final, aunque anuncia conclusiones, es más bien un compendio 
de elementos no desarrollados en el resto del libro así como una discusión obligada del trabajo de Thomas Piketty con respecto a la desigualdad.

Habiendo reseñado la dimensión metodológica que se trabaja a lo largo del libro, y ante la imposibilidad de desarrollar cada capítulo, me detendré ahora en tres comentarios específicos que abordan temas que considero relevantes desde una mirada de economía política contemporánea.

El primero es sobre su teoría del comercio y los términos de intercambio reales, que desarrolla en el capítulo once y que construye sobre su trabajo previo al respecto de los efectos de la globalización. Aquí señala que la teoría económica ortodoxa se sostiene en dos premisas problemáticas que provienen de la teoría clásica de David Ricardo: (1) que el libre cambio está regulado por las ventajas (costos) comparativas y (2) que este lleva al pleno empleo en las naciones participantes. El mecanismo supuesto para que se den estas premisas está en la fluidez con el que se equilibran y balancean los tipos de cambio reales con las balanzas comerciales, distribuyendo óptimamente el comercio y el empleo. Frente al hecho empírico que muestra rigidices o "problemas de elasticidad" en estas transiciones, tanto la teoría ortodoxa (hablando de que el balanceo se da solo en el largo plazo) como la heterodoxa (incorporando oligopolios, economías de escala, etc.) han tratado de enmendar los fallos de la teoría, pero para Shaikh la única solución pasa por rehacer la teoría desde su inicio, lo que implica renunciar a las premisas y al modelo de Ricardo.

El punto es que el modelo de Ricardo comete un error capital al considerar que las mercancías fluyen completamente separadas de los flujos financieros; es decir, no articula correctamente la balanza comercial con la balanza de pagos. Reparando en aquello, Shaikh propone que su enfoque clásico señalará la centralidad de las ventajas o costos absolutos como determinantes del comercio (su dirección, fundamentalmente). En primer lugar, debido a la ya mencionada relación entre precios y costos, eventualmente la competitividad estará atada a las diferencias de eficiencia, de salarios reales, y de proporciones técnicas de los países, determinantes que los cambios en los términos internacionales o desbalances comerciales podrán influir, pero no alterar lo suficiente. En segundo lugar, los flujos de capitales interrumpen las supuestas lógicas de balanceo que se suponen en el modelo estándar. Entonces, el tipo de cambio real estará influido por las estructuras de costos (que implican la productividad y los salarios) de las economías comerciantes, así como la tasa de bienes transables y no transables, lo que nos sugiere que no tienden necesariamente a un comercio balanceado, a menos que las economías sean muy similares.

El acervo empírico que Shaikh recopila muestra la persistencia del desbalance comercial en todo el mundo. Esto implica que la hipótesis de la paridad en poder adquisitivo no se sostiene (con excepción de momentos de alta inflación). Tal argumento teórico ha permitido a Shaikh, en publicaciones previas, plantear una crítica contra las políticas neoliberales de globalización y libre comercio, sobre todo en relación con el tercer 
mundo. Actualmente, el argumento también puede usarse para pensar los problemas a los que se enfrenta Estados Unidos en relación con sus términos de intercambio y el empleo, que ha tenido repercusión en el ciclo político con consecuencias aún no claras.

El segundo punto es con respecto a la teoría macroeconómica de Shaikh, que tiene entre sus intenciones responder al hecho que el capitalismo, bajo un esquema de salarios reales flexibles, produce una tasa persistente de desempleo (contradiciendo así tanto la teoría neoclásica, como también la keynesiana que permite una eliminación de este con estimulación fiscal y monetaria). Para esto recupera el argumento de Marx con respecto al "ejército industrial de reserva", que luego Richard Goodwin formalizaría en un modelo dinámico (inspirado en las ecuaciones Lotka-Volterra, hechas para modelar la dinámica de la interacción de dos poblaciones de animales: depredadores y presas), observando cómo la organización de trabajadores para negociar los salarios (algo presente en Keynes) es esencial para pensar las relaciones del salario con el desempleo. La manera en que Shaikh las formaliza (distinta a Goodwin) permite la confección de una "curva clásica" que relaciona de manera negativa la variación en la participación de los salarios con el desempleo. Esto permite observar dos cosas: 1) la existencia de un punto de estabilidad del cambio en la participación de los salarios que se condice con una tasa positiva de desempleo; 2) la posibilidad que la derrota de la organización obrera habilite una caída en el desempleo debido al aumento de rentabilidad. Esto permitiría modelar la existencia no solo del desempleo persistente, sino también la manera en que los intentos de llegar al pleno empleo son derrotados por la lógica misma del sistema.

Hacia el final del capítulo catorce, así como en el siguiente capítulo, Shaikh trabajará la relación de esta "curva clásica" con la curva de Phillips. Aunque el objetivo de ambas relaciones es la misma (pensar los determinantes del desempleo), la curva clásica no relaciona el desempleo con la inflación, sino con la disputa entre el capitalista y el trabajo (lo que en el fondo es la rentabilidad nuevamente), lo que quiere decir que la curva debe su forma a una determinación social (no técnica). Sin embargo, la curva clásica grafica una relación similar a la curva de Phillips, manteniéndose incluso después de su destitución empírica tras la crisis de estanflación, ya que Shaikh muestra cómo, tras desdibujarse en los años setenta (el ataque al poder de negociación de los trabajadores), la curva clásica se reconstituye nuevamente a nivel más bajo que en el periodo previo. Esto se articula con su teoría clásica de la inflación, que se encuentra relacionada directamente con el nivel de precios y la rentabilidad más que con el desempleo, siendo esencial un enfoque combinado de demanda (pull) y oferta (push) que teorice la inflación en relación con la ratio de capacidad utilizada (la impronta clásica de este argumento se puede rastrear hasta el modelo de maíz de Ricardo o los esquemas de reproducción de Marx). Por otro lado, Shaikh trata de señalar cómo su argumento de una tasa normal de desempleo no es similar a la tasa de paro con inflación estable (la NAIRU) de Friedman-Phelps, ya que además de dar un lugar al efecto del dinero en mover el desempleo (hasta el límite de la capacidad potencial), este desempleo no surge por una fricción en los tiempos de 
re-contratación o un límite técnico, sino que es producto del capitalismo en su óptimo funcionamiento en competencia real.

Esta teoría del desempleo se muestra muy relevante a la luz de los desarrollos posteriores a la crisis del 2008 con respecto a las recuperaciones débiles con poca generación de empleo. Shaikh muestra una explicación sistémica y endógena de este fenómeno, que además es rastreable históricamente. Más intrigantes, sin embargo, son las consecuencias de políticas de este enfoque, que considera insuficientes las propuestas de las perspectivas keynesianas y post-keynesianas (contra la austeridad, por políticas expansivas). Visto de cierta manera, debido a su complejidad y endogenización de una gran mayoría de variables en otros modelos independientes, su sistema parece determinista respecto a la agencia política. Eso puede leerse de una manera pesimista, entendiendo que no hay forma de resolver prontamente los problemas del capitalismo, o más bien encerrando una llamada más radical que la simple policy, haciendo imaginables reformas estructurales en el sentido socialista del término ante la debacle social a la que se enfrenta el futuro de la economía.

Mi último comentario se refiere al apartado final del libro, donde figuran unos cuántos párrafos dedicados a la relación del mundo desarrollado con el mundo en desarrollo. Aquí Shaikh busca justificar el haberse enfocado en el primer mundo a lo largo del libro a través de sostener que esto nos permitirá entender el mundo en desarrollo y que los patrones recurrentes del capitalismo trascienden las diferencias geográficas y sociales. Esta reflexión es importante porque, si consideramos cierta su teorización del capitalismo avanzado, se evitarán los maniqueísmos exagerados entre un mundo desarrollado de "libre mercado", con derechos de propiedad claros que lo hacen productivo; y un mundo en desarrollo carente de todas aquellas cualidades. Estas caracterizaciones son peligrosas porque ofrecen recetas equivocadas para el desarrollo (modernización liberal, tratados de libre comercio, eliminación del estado desarrollista o regulador, etc.), que ni siquiera fueron la razón del progreso en el centro, sino más bien la idealización creada por la teoría neoclásica.

Sin embargo, a pesar de esta clarificación final, sorprende que las refracciones del capitalismo en otras latitudes no sean modeladas con el mismo interés que el centro. En un libro reciente, similar en el objetivo y ambición que el de Shaikh, el economista peruano Adolfo Figueroa (Growth, Employment, Inequality and Environment: The Unity of Knowledge in Economics) ha representado el capitalismo como el sistema dinámico compuesto por la relación de tres tipos de sociedades capitalistas (primer mundo, países en desarrollo sin herencia colonial y países en desarrollo con herencia colonial), mostrando también cómo el motivo de la búsqueda de ganancias es la motivación de su mecánica. Un diálogo entre ambas propuestas puede ser enriquecedor. La obra de Shaikh se beneficiaría de un modelo de interrelación del centro y la periferia que permite además modelar el sub-empleo, cada vez más importante, no solo en el tercer mundo, y que no asume los supuestos de la teoría neoclásica. Asimismo, el trabajo 
de Figueroa también puede aprovechar de Shaikh su revaloración del modelo clásico (el cual Figueroa descarta rápidamente) para explicar la mayoría de hechos estilizados claves del capitalismo histórico.

En suma, estos son algunos comentarios de la inmensa cantidad que se pueden hacer desde la obra de Anwar Shaikh - se queda en el tintero abordar la revisión de la teoría del valor trabajo o su análisis sobre los ciclos Kondratieff y su influencia en la crisis actual - , lo que atestigua su envergadura. Sin embargo, es importante señalar que este libro, desde su densidad, llama a ser utilizado para elaborar libros de textos y programas de investigación que permitan hacer operativa su propuesta teórica. Ese es el objetivo implícito del libro y también tendrá que ser evaluado por el éxito que logre al respecto (algo que ya no dependerá tanto del autor).

Stephan Gruber

Departamento de Economía

Pontificia Universidad Católica del Perú 